\title{
On the Kinematics and Synthesis of the Geared Five-Bar Slider-Crank Mechanism
}

\author{
Ibrahim A. Sultan and Azfar Kalim \\ School of Science and Engineering, \\ University of Ballarat, \\ PO Box 663, \\ Ballarat VIC 3553, Australia.
}

\begin{abstract}
The geared five-bar mechanism possesses kinematic abilities which qualify it for use in various industrial applications. Small changes to the mechanism topology or dimensions create new designs with different motion characteristics. This paper presents designorientated kinematical insights and mathematical treatments for the embodiment of the mechanism in which the end gear is eccentrically pivoted to a sliding element. For the synthesis effort, a kinematic classification will be introduced and approximate curves will be used to guide the motion of the slider. A gradient-based Levenberg-Marquardt formulation will be employed for the optimisation procedure. Geometric, mobility and dimensional constraints will be utilised together with numerical position equations for the analysis. Two case studies are presented at the end of the paper to highlight the versatility of the mechanism and prove the validity of the presented mathematical model.
\end{abstract}

Keywords: Kinematic - Mechanism - Synthesis - Gears - Optimisation - Slider Levenberg - Marquardt

\section{INTRODUCTION}

As suggested by Gabriele (1993), the availability of computing resources and optimisation techniques has motivated effort to design linkages capable of following curves and functions. However, the application of these techniques has also highlighted 
some mathematical difficulties pertinent to the field of linkage synthesis. For example, the geometric constraints of a given mechanism may limit its ability to follow a desired path; or a non-assembly solution may occur during the course of iterative procedures. Cossalter et (1992) explain that these situations often result in numerical instabilities. In fact, non-assembly has been given a special attention by a number of researchers including Minnaar et al (2001) who used multi-body formulation and gradient-based optimisation technique for mechanism synthesis. An approach which has also been utilised in the work by Jensen and Hansen (2006). Earlier however, Hansen (2002) had presented a method which featured the insertion of fictitious joints and links on the mechanism structure to increase its mobility during the optimisation procedure. This is likely to reduce numerical instabilities which result from non-assembly. Zhang et al (1998) present an interesting paper which features direct application of the optimisation theory in the field of mechanism synthesis. In their paper, an algorithm has been developed based on the convex problem formulation to design mechanisms capable of tracing a set of design points. The work by Dooner (2001) features the combination of two four-bar chains in a fashion that will produce a degree of mobility suitable for pathfollowing applications.

Cabrera et al (2002) and Ded and Tiwari (2005) use Genetic Algorithms (GA) to solve the synthesis problem. Tabu search is another simulation-based search technique which has successfully been utilised by Smaili et al (2005) for mechanism design.

A geared five-bar mechanism has been synthesised by Nokleby and Podhorodeski (2001) who employed Grashoff's condition and an elegant technique to transform the constrained optimisation problem into a non-constraint one. The papers of Suchora and Savage (1974a and 1974b) represent an in depth effort in the area of five-bar geared mechanisms. Whilst the focus of their work was the version of the mechanism in which the first gear is stationary, the focus in the present paper is the design in which the last gear is eccentrically pivoted to a sliding element as schematically depicted in figure (1) below. A version of this embodiment was patented by Bresland (2001) as a drive mechanism for internal combustion engines. However, it should be pointed out here that 
despite the outstanding kinematical flexibility this design offers, it does feature a gear-set pivoted on a floating arm. Whilst such an arrangement may be acceptable for low speed applications, it does place a caveat on high speed utilisation of the mechanism due to inertia loading and associated vibration effects. It would be advisable to weigh these dynamical aspects against the gains hoped to be achieved from the mechanism kinematics before a decision is made on whether or not to adopt the mechanism for a specific application. A decision which should be carefully made in the light of such factors as the density and durability of available materials, the ability to control the effects of inertia forces and the expected quality of the manufacturing process. This paper is dedicated to study the kinematics and synthesis of the mechanism which are worthy of being investigated on their own right as will be made evident by the various sections of the paper.

\section{Position EQUATIONS}

As shown in figure (1), the geared five-bar slider-crank mechanism consists of an epicyclical gear train installed on a floating arm. The first gear (number 1) in the set is pivoted off-centre to the origin of a Cartesian frame, and the last gear (number $n$ ) is also pivoted eccentrically to a sliding member. Whilst the arm length is given as $l_{a}$, the distance from centre to pivot on the first and last gears are $r_{c 1}$ and $r_{c n}$ respectively. If these two distances need to be greater than the physical dimensions of the first and last gears, extensions may be rigidly connected to the gears as shown in figure (1). The overall gear ratio, $\beta$, of the set is given as follows;

$\beta==(-1)^{n-1} \frac{D_{1}}{D_{n}}$

where $D_{1}$ and $D_{n}$ are the pitch circle diameters of the first gear and the last gear respectively.

For an epicyclical gear train, Norton (1999) presents the following velocity relationship; 


$$
\beta=\frac{\left(\frac{d \psi}{d t}-\frac{d \mu}{d t}\right)}{\left(\frac{d \theta}{d t}-\frac{d \mu}{d t}\right)}
$$

where $\theta, \mu$ and $\psi$ are the angular displacements of the crank (i.e. the first gear), the arm and the last gear respectively. Figure (1) indicates the manner in which these angles are measured in the context of the work presented here. In equation (2), $\frac{d \bullet}{d t}$ signifies differentiation with respect to time. This equation can be manipulated as follows;

$$
\frac{d \psi}{d \theta}=(1-\beta) \frac{d \mu}{d \theta}+\beta
$$

Employing the concept of loop-closure, the instantaneous $\mathrm{x}$-coordinate of the slider pivot, $x$, and its constant y-coordinate, $h$, can be expressed as follows;

$x=r_{c 1} \cos \left(\theta_{s}+\theta\right)+l_{a} \cos \left(\mu_{s}+\mu\right)-r_{c n} \cos \left(\psi_{s}+\psi\right)$

and

$h=r_{c 1} \sin \left(\theta_{s}+\theta\right)+l_{a} \sin \left(\mu_{s}+\mu\right)-r_{c n} \sin \left(\psi_{s}+\psi\right)$

The initial values of the link angles, $\theta_{s}, \mu_{s}$ and $\psi_{s}$ are defined by the synthesis procedure and used to position the various links during assembly.

\section{Kinematic ClaSsificAtion}

Without loss of generality, the three angular displacements $\theta, \mu$ and $\psi$ may all be set equal to zero at the assembly position. As such equation (3) can be integrated as follows;

$\psi=(1-\beta) \mu+\beta \theta$ 
Two possible design variations of the geared five-bar slider-crank mechanism have been identified and classified in this paper to facilitate future work in this area. These two variations are referred to here as Type 1 and Type 2 mechanisms. The Type 1 design is dimensioned in such a fashion that the arm can only rock through an angular span and the last gear can perform continuous rotations. On the other hand, the Type 2 mechanism is designed so that the arm can perform continuous rotations and the last gear can only perform rocking strokes. As suggested by the synthesis procedure presented later in the paper, a constraint may be employed to relate the three lengths, $l_{a}, r_{c 1}$ and $r_{c n}$ in order for the optimisation procedure to yield either a Type 1 design or a Type 2 design as desired. Figure (2) presents sample Type 1 and Type 2 mechanisms which can be synthesised to yield the same slider motion.

In a Type 1 mechanism, the arm exhibits rocking motion where the displacement $\mu$ fluctuates about the value of zero. This value is set at the assembly position (i.e. at the start of motion) and recurs at the start of each cycle. Along with all other displacements, $\psi$ is equal to zero at the start of motion, but at the end of the first cycle $\psi$ is calculated from equation (6) as equal to $2 \pi \beta \sigma$, where the product $\beta \sigma$ is an integer. In fact, $\sigma$, which signifies the number of rotations performed by the crank during one work cycle, is the smallest integer which makes $\beta \sigma$ also an integer. To calculate $\sigma$, the gear ratio $\beta$ has to be expressed in a simplified fractional form rather than a decimal form (e.g. $2 / 3$ rather than 0.667).

In a Type 2 mechanism, the last gear exhibits rocking motion where the displacement $\psi$ fluctuates about the value of zero. This value is set at the start of motion (i.e. the assembly position) and recurs at the start of each cycle. Along with all other displacements, $\mu$ is equal to zero at the start of motion, but at the end of the first cycle it is calculated from equation (6) as equal to $2 \pi \beta \sigma /(\beta-1)$, where $\beta \neq 1$ and the product $\beta \sigma /(\beta-1)$ is an integer. In this case, $\sigma$ which still signifies the number of rotations performed by the crank during one work cycle, is the smallest integer which makes the product $\beta \sigma /(\beta-1)$ also an integer. Designs in which $\beta$ is set equal to unity 
produce Type 1 mechanisms since equation (6) will reduce to $\psi=\theta$. However, such mathematical simplicity is likely to reduce the ability of the mechanism to conform to some special stroke requirements if stipulated by the designer.

\section{NuMERICAL POSITION SOLUTION}

In the context of the work presented here, position analysis is a differential-model-based numerical procedure carried out to calculate the positions of various links against infinitesimal progression of the crank displacement. The differential model of the geared five-bar slider-crank mechanism can be obtained by differentiating equations (4) and (5) with respect to $\theta$ as follows;

$\frac{d x}{d \theta}=-r_{c 1} \sin \left(\theta_{s}+\theta\right)-l_{a} \sin \left(\mu_{s}+\mu\right) \frac{d \mu}{d \theta}+r_{c n} \sin \left(\psi_{s}+\psi\right) \frac{d \psi}{d \theta}$

and

$0=r_{c 1} \cos \left(\theta_{s}+\theta\right)+l_{a} \cos \left(\mu_{s}+\mu\right) \frac{d \mu}{d \theta}-r_{c n} \cos \left(\psi_{s}+\psi\right) \frac{d \psi}{d \theta}$

The set of ordinary differential equations (3), (7) and (8) can now be solved numerically using Euler forward method. For this procedure, the cyclical motion of the crank is divided in $\mathrm{L}$ infinitesimal jumps; where $\mathrm{L}$ is selected large enough to ensure a desired level of accuracy. At step number $i$ (where $i=0 \cdots L-1$ ), displacements, $\delta x_{i}, \delta \mu_{i}$ and $\delta \psi_{i}$ which correspond to a given value of the infinitesimal displacement, $\delta \theta$ (where $\delta \theta=2 \pi \sigma / L)$ can be obtained as follows;

$\mathbf{J}_{\theta i}\left[\begin{array}{c}\delta x_{i} \\ \delta \mu_{i} \\ \delta \psi_{i}\end{array}\right]=\delta \theta\left[\begin{array}{c}r_{c 1} \sin \left(\theta_{s}+\theta_{i}\right) \\ -r_{c 1} \cos \left(\theta_{s}+\theta_{i}\right) \\ -\beta\end{array}\right]$ 
where $\theta_{i}$ is the value of the crank displacement, $\theta$, at step number $i$ and the kinematic Jacobian, $\mathbf{J}_{\theta i}$, is expressed as follows;

$$
\mathbf{J}_{\theta i}=\left[\begin{array}{ccc}
-1 & -l_{a} \sin \left(\mu_{s}+\mu_{i}\right) & r_{c n} \sin \left(\psi_{s}+\psi_{i}\right) \\
0 & l_{a} \cos \left(\mu_{s}+\mu_{i}\right) & -r_{c n} \cos \left(\psi_{s}+\psi_{i}\right) \\
0 & 1-\beta & -1
\end{array}\right]
$$

where $\mu_{i}$ and $\psi_{i}$ are the values of $\mu$ and $\psi$ respectively at step number $i$. As pointed out by Haug (1989), the condition that the mechanism will not run into a singularity during motion can simply be stated as follows;

$$
\left|\mathbf{J}_{\theta i}\right|=l_{a} \cos \left(\mu_{s}+\mu_{i}\right)-r_{c n}(1-\beta) \cos \left(\psi_{s}+\psi_{i}\right) \neq 0
$$

It is obvious from the above equation that if $\beta$ was set equal to unity, the condition for non-singularity is $\pi / 2>\mu_{s}+\mu>-\pi / 2$, which suggests a Type 1 mechanism with a rocking arm as has been concluded earlier. Equation (11) also suggests that the determinant of $\mathbf{J}_{\theta i}$ has to remain either positive or negative during the whole course of motion. A change of sign will imply that the value of zero has been crossed over in the transition.

The updated values of the link total displacements at the start of step number $i+1$ can be calculated as follows;

$$
\left.\begin{array}{c}
\theta_{i+1}=\theta_{i}+\delta \theta_{i} \\
x_{i+1}=x_{i}+\delta x_{i} \\
\mu_{i+1}=\mu_{i}+\delta \mu_{i} \\
\psi_{i+1}=\psi_{i}+\delta \psi_{i}
\end{array}\right\}
$$

To find a mathematical condition to determine as to whether the slider is at one of its stationary positions, it is possible to re-arrange equation (9) in the following form; 


$$
\mathbf{J}_{x i}\left[\begin{array}{c}
\delta \theta \\
\delta \mu_{i} \\
\delta \psi_{i}
\end{array}\right]=\left[\begin{array}{c}
\delta x_{i} \\
0 \\
0
\end{array}\right]
$$

where the matrix $\mathbf{J}_{x i}$ is another kinematic Jacobian for the mechanism, and it can be expressed as follows;

$$
\mathbf{J}_{x i}=\left[\begin{array}{ccc}
-r_{c 1} \sin \left(\theta_{s}+\theta_{i}\right) & -l_{a} \sin \left(\mu_{s}+\mu_{i}\right) & r_{c n} \sin \left(\psi_{s}+\psi_{i}\right) \\
r_{c 1} \cos \left(\theta_{s}+\theta_{i}\right) & l_{a} \cos \left(\mu_{s}+\mu_{i}\right) & -r_{c n} \cos \left(\psi_{s}+\psi_{i}\right) \\
\beta & 1-\beta & -1
\end{array}\right]
$$

For the slider to be stationary, the determinant of the matrix $\mathbf{J}_{x i}$ has to vanish. At step number $i$ this determinant can be simplified as follows;

$$
\begin{aligned}
\left|\mathbf{J}_{x i}\right| & =l_{a} r_{c n} \beta \sin \left(\mu_{s}+\mu_{i}-\psi_{s}-\psi_{i}\right)-l_{a} r_{c 1} \sin \left(\mu_{s}+\mu_{i}-\theta_{s}-\theta_{i}\right) \\
& +r_{c n} r_{c 1}(1-\beta) \sin \left(\psi_{s}+\psi_{i}-\theta_{s}-\theta_{i}\right)
\end{aligned}
$$

The next section offers an insight into the mechanism synthesis technique used in this paper.

\section{MECHANISM SYNTHESIS}

To synthesise a geared five-bar slider-crank mechanism a numerical procedure is adopted to calculate the design vector, $\left[\begin{array}{llllll}\theta_{s} & \mu_{s} & \psi_{s} & r_{c 1} & r_{c n} & l_{a}\end{array}\right]^{T}$. It should be noted that $h$ has not been included in the design vector since it can be calculated by re-writing equation (5) at the assembly position, where all displacements are set equal to zero, as follows;

$$
h=r_{c 1} \sin \left(\theta_{s}\right)+l_{a} \sin \left(\mu_{s}\right)-r_{c n} \sin \left(\psi_{s}\right)
$$


Usually, for each stroke, the starting and end conditions are given in terms of $\mathrm{x}$ coordinates of the slider at stationary points. If this is taken into account, the premise in this paper is that it possible to suggest any smooth non-fluctuating mathematical curve to roughly approximate the slider motion between stationary points as a function of the crank angle, $\theta$. Examples of mathematical constructs which can be utilised by the designer to create these curve are the cosine functions, the cycloidal functions and cubic splines. On the resulting curves, the $M$ stationary timing points, at which the slope vanishes, correspond to the ends of the strokes. These timing terminals are chosen as precision points at which high weighting values will be given to both the distance-based objective functions and velocity constraints. A number of intermediate points on the curves are then chosen and used only to "guide" the direction in which the slider should move. Accurate matching of these "guiding" points is not required; and as such no preference is given in this paper to any particular mathematical construct used to generate them. In the procedure presented in this paper, the total number of points chosen on the approximate guiding curve is $N$; and the points for every stroke can be spaced out either equally or by using a Chebyshev series. Figure (3) depicts a conceptual representation of the guiding curve.

The structural error is utilised in this paper as the objective function, $f_{x q}$, which should be minimised. This function is expressed in reference to equation (5) as follows;

$$
f_{x q}=r_{c 1} \cos \left(\theta_{s}+\theta_{q}\right)+l_{a} \cos \left(\mu_{s}+\mu_{q}\right)-r_{c n} \cos \left(\psi_{s}+\psi_{q}\right)-x g_{q}
$$

where $q=0 \cdots N-1$ and $x g_{q}$ is the x-coordinate of point number $q$ on the guiding curve.

The minimisation process is subject to the equality constraints, $g_{v j}$ (where $j=0 \cdots M-1)$, which are introduced to ensure compliance with stroke timing requirements as follows; 


$$
\begin{aligned}
g_{v j}= & l_{a} r_{c n} \beta \sin \left(\mu_{s}+\mu_{j}-\psi_{s}-\psi_{j}\right)-l_{a} r_{c 1} \sin \left(\mu_{s}+\mu_{j}-\theta_{s}-\theta_{j}\right) \\
& +r_{c n} r_{c 1}(1-\beta) \sin \left(\psi_{s}+\psi_{j}-\theta_{s}-\theta_{j}\right)=0
\end{aligned}
$$

The optimised solution is also subject to the following mobility constraint;

$$
\prod_{q=0}^{N-1}\left[l_{a} \cos \left(\mu_{s}+\mu_{q}\right)-r_{c n}(1-\beta) \cos \left(\psi_{s}+\psi_{q}\right)\right]>0
$$

where $N-1$ is assumed to be an odd number. However, $N-1$ is usually calculated by dividing the cyclical angular displacement of the crank by a desired angular interval. Thus, if $N-1$ has been found to be even at the start of the numerical procedure, the consecutive multiplications in equation (19) will be taken only up to $N-2$ terms in order to ensure a positive product.

The following three constraints have been introduced to impose minimum or maximum limits on dimensions;

$\left(r_{c 1} \sin \left(\theta_{s}\right)+l_{a} \sin \left(\mu_{s}\right)-r_{c n} \sin \left(\psi_{s}\right)\right)^{2}-h_{\max }^{2} \leq 0$

$r_{c 1}-r_{c 1 \min } \geq 0$

and

$$
r_{c n}-r_{c n \min } \geq 0
$$

where $h_{\max }, r_{c 1 \text { min }}$ and $r_{c n \text { min }}$ are positive numbers employed to signify limits imposed on their respective dimensions.

Inequality (22) is used only for Type 1 mechanisms; for a Type 2 mechanism this inequality is replaced by $l_{a}-l_{a \text { min }} \geq 0$, where $l_{a \text { min }}$ is the minimum allowable arm length. Moreover, the following constraint has been employed to make sure that the arm length is large enough to ensure limited angular stroke for the arm in a Type 1 mechanism; 
$l_{a}-\sqrt{r_{c 1}^{2}+r_{c n}^{2}} \geq 0$

where for a Type 2 mechanism, this inequality is replaced by $r_{c n}-\sqrt{r_{c 1}^{2}+l_{a}^{2}} \geq 0$.

Antoniou and $\mathrm{Lu}$ (2007) explain that that inequality constraints can be transformed into equality constraint functions, $g_{m}, g_{h}, g_{c 1}, g_{c n}$ and $g_{l}$, by introducing a set of "slack variables" as follows;

$$
\left.\begin{array}{l}
g_{m}=-a_{m}^{2}+\prod_{q=0}^{N-1}\left[l_{a} \cos \left(\mu_{s}+\mu_{q}\right)-r_{c n}(1-\beta) \cos \left(\psi_{s}+\psi_{q}\right)\right]=0, \\
g_{h}=\left(r_{c 1} \sin \left(\theta_{s}\right)+l_{a} \sin \left(\mu_{s}\right)-r_{c n} \sin \left(\psi_{s}\right)\right)^{2}-h_{\max }^{2}+a_{h}^{2}=0, \\
g_{c 1}=r_{c 1}-r_{c 1 \min }-a_{c 1}^{2}=0, \\
g_{c n}=r_{c n}-r_{c n \min }-a_{c n}^{2}=0 \quad \text { and } \\
g_{l}=l_{a}-\sqrt{r_{c 1}^{2}+r_{c n}^{2}}-a_{l}^{2}=0
\end{array}\right\}
$$

The slack variables, $a_{m}, a_{h}, a_{c 1}, a_{c n}$ and $a_{l}$, featured in (24) will result in an augmented design vector $\mathbf{v}$, where $\mathbf{v}=\left[\begin{array}{lllllllllll}\theta_{s} & \mu_{s} & \psi_{s} & r_{c 1} & r_{c n} & l_{a} & a_{m} & a_{h} & a_{r c 1} & a_{r c n} & a_{l}\end{array}\right]^{T}$. It is worthy of noting here that the set of equations in (24) is written for a Type 1 mechanism. For a Type 2 mechanism, the bottom two equations in the set are replaced by the following;

$$
\left.\begin{array}{l}
g_{c n}=r_{c n}-\sqrt{r_{c 1}^{2}+l_{a}^{2}}-a_{c n}^{2}=0 \\
\text { and } \\
g_{l}=l_{a}-l_{a \min }-a_{l}^{2}=0
\end{array}\right\}
$$

The next section presents the details of the mathematical implementation of the mechanism synthesis model.

\section{Computational Procedure}

In the model presented here, the error vector, $\mathbf{e}$ is given, in relation to the expressions detailed in (17), (18), (24) and (25), as follows; 
$\mathbf{e}=\left[\begin{array}{lllllllllll}f_{x 0} & \cdots & f_{x N-1} & g_{v 0} & \cdots & g_{v M-1} & g_{m} & g_{h} & g_{r c 1} & g_{r c n} & g_{l}\end{array}\right]^{T}$

During iterations, e is updated and employed to calculate the design vector correction, $\delta \mathbf{v}$.

The gradient-based optimisation technique employed in this paper features a LevenbergMarquardt formulation. This robust method is suitable for applications with inherent mathematical singularities such as mechanism synthesis. As shown by equation (27) below, the method is equipped with a positive real damping factor, $\lambda$, introduced to ensure a slow progression away from singular points and then accelerate the convergence rate near the solution points. This process involves the use of the system Jacobian, $\mathbf{J}$, whose entries are the partial derivatives, of the functions which constitute the vector $\mathbf{e}$.

As summarised by the flowchart given in Figure (4), convergence is assessed at iteration number $k$ by the most updated error vector, $\mathbf{e}_{k}$ (i.e. $\left\|\mathbf{e}_{k}\right\| \leq \varepsilon$, where $\|\bullet\|$ indicates the Euclidean norm and $\varepsilon$ is a small positive number). If convergence has not been achieved, the iterative process continues using the following expression;

$\delta \mathbf{v}_{k}=\left(\mathbf{J}_{k}^{T} \mathbf{W} \mathbf{J}_{k}+\lambda \mathbf{I}\right)^{-1} \mathbf{J}_{k}^{T} \mathbf{W} \mathbf{e}_{k}$

where $\mathbf{J}_{k}$ and $\delta \mathbf{v}_{k}$ are the current values of $\mathbf{J}$ and $\delta \mathbf{v}$, respectively. The design vector is subsequently updated (i.e. $\mathbf{v}_{k+1}=\mathbf{v}_{k}-\delta \mathbf{v}_{k}$ ) and the kinematic analysis is performed using the numerical procedure described in equations (9) to (12).

In equation (27), $\mathbf{W}$ is a diagonal matrix whose positive entries are chosen for every application to reflect the weighting which every function should have on the resulting solution. This matrix has been used to distinguish the stationary points (i.e. precision points) from the intermediate points which are only used to guide the direction of the slider motion. The identity matrix, $\mathbf{I}$, in equation (27) is of dimension $11 \times 11$ and $\lambda$ is 
initially assigned a large value. This value is then progressively reduced during iterations to regain the favourable convergence aspects of the Newton iterative technique. The rate at which $\lambda$ is reduced during the iterations has to be fairly slow in order for useful solution points to be captured by the procedure. This is needed to deal with the mathematical aspects of the mechanism synthesis problems, which often suffer from singularities and non-assembly complications. Case studies are presented in the next section.

\section{CASE STUdies}

In this section, two design case studies are presented to illustrate the kinematical focus featured in the paper. For each case study, the number of iterations, in which a solution has been found, will be given along with the associated structural error as defined by equation (17). It is worthy of noting here that the method employed for the analysis, being gradient in nature, is possible to produce a local minimum solution rather than a global one. As such, the results obtained are likely to be influenced by the initial guess values assigned to the design parameters. Moreover, initial guess values which are considerably far from the target solutions may produce numerical singularities unless the damping factor, $\lambda$, is reduced very slowly during the iterative procedure. If this still does not improve the numerical stability of the iterations, new guess values should be attempted. Experience suggests that many sets of successful guess values will quickly be found for the solution.

\section{CASE STUDY 1}

This case study features the utilisation of the geared five-bar slider-crank mechanism to produce strokes of different lengths in the one cycle. For the case study, the guiding curve has been synthesised using cosine functions over two crank rotations (i.e. $\sigma=2$ ) to reflect the motion particulars given in Table 1 below. It is required to design a Type 1 mechanism and the gear ratio, $\beta$, was set equal to $1 / 2$ to ensure one end gear revolution per cycle. 
Table 1. The desired stroke particulars for Case Study 1

\begin{tabular}{|l|c|c|c|c|}
\hline Stroke Number & 1 & 2 & 3 & 4 \\
\hline x-coordinate at start of stroke (mm) & 114 & 250 & 214 & 250 \\
\hline x-coordinate at end of stroke (mm) & 250 & 214 & 250 & 114 \\
\hline Corresponding crank rotation (deg) & 215 & 145 & 145 & 215 \\
\hline
\end{tabular}

A Chebyshev series was used to guide the slider motion; and the initial guess dimensions were given as follows;

$\theta_{s}=-90^{\circ}, \psi_{s}=0^{\circ}, \mu_{s}=0^{\circ}, r_{c 1}=20 \mathrm{~mm}, r_{c n}=20 \mathrm{~mm}$ and $l_{a}=190 \mathrm{~mm}$

At the end of iterations, the produced slider trajectory is shown in figure (5) along with the guiding points used for the analysis. The figure also shows the trajectory which corresponds to the initial values used for design parameters. The resulting design parameters have been calculated as;

$\theta_{s}=180.57^{\circ}, \psi_{s}=0.51^{\circ}, \mu_{s}=0.42^{\circ}, h=0.67 \mathrm{~mm}, r_{c 1}=37.07 \mathrm{~mm}, r_{c n}=49.49 \mathrm{~mm}$ and $l_{a}=202.55 \mathrm{~mm}$.

The optimised solution has been found after 292 iterations, and the mean value of the structural error and its standard deviation have been calculated, respectively, as $-1.447 \mathrm{~mm}$ and $1.918 \mathrm{~mm}$.

\section{CASE STUDY 2}

This case study features a mechanism with a single revolution cycle (i.e. $\sigma=1$ ) and two equal stokes separated by a dwell. The guiding curve has been synthesised using cosine functions to reflect the motion particulars given in Table 2. The two strokes given in the table are to be separated by $7.5^{\circ}$ dwell. It is required to design a Type 1 mechanism with a gear ratio, $\beta$, set equal to -1 to ensure one revolution per cycle by the last gear. 
Table 2. The desired stroke particulars for Case Study 2

\begin{tabular}{|l|c|c|}
\hline Stroke Number & 1 & 2 \\
\hline X-coordinate at start of stroke $(\mathrm{mm})$ & 100 & 200 \\
\hline X-coordinate at end of stroke $(\mathrm{mm})$ & 200 & 100 \\
\hline Corresponding crank rotation $(\mathrm{deg})$ & 147.5 & 205 \\
\hline
\end{tabular}

A Chebyshev series was used to guide the slider motion; and the initial guess dimensions were given as follows;

$\theta_{s}=90^{\circ}, \psi_{s}=90^{\circ}, \mu_{s}=0^{\circ}, r_{c 1}=20 \mathrm{~mm}, r_{c n}=20 \mathrm{~mm}$ and $l_{a}=200 \mathrm{~mm}$

At the end of iterations, the slider trajectory was produced as depicted in figure (6) along with the guiding points used for the analysis. The figure also shows the trajectory which corresponds to the initial guess values given for design parameters. The resulting design parameters have been found as follows;

$\theta_{s}=-24.49^{\circ}, \psi_{s}=151.37^{\circ}, \quad \mu_{s}=9.33^{\circ}, \quad h=-4.96 \mathrm{~mm}, \quad r_{c 1}=33.66 \mathrm{~mm}, \quad r_{c n}=30.20 \mathrm{~mm}$ and $l_{a}=144.77 \mathrm{~mm}$.

The optimised solution has been found after 553 iterations, and the mean value of the structural error and its standard deviation have been calculated, respectively, as $-1.17 \mathrm{~mm}$ and $2.34 \mathrm{~mm}$.

\section{CONCLUSIONS}

The geared five-bar slider-crank mechanism can be designed to produce desirable stroke characteristics required for various applications. This paper presents a kinematical description of the mechanism along with a suitable synthesis model. The model features the numerical position equations together with the objective functions and constraints used for the synthesis process. Approximate curves have been used to guide the motion of the slider, and a gradient-based Levenberg-Marquardt formulation has been employed for the optimisation procedure. The case studies presented at the end of the paper highlight the versatility of the mechanism and prove the validity of the presented mathematical model. 


\section{Acknowledgments}

The authors would like to express their deepest appreciation to the reviewers for their outstanding comments and insight.

\section{Nomenclature}

$l_{a} \quad$ arm length

$r_{c 1} \quad$ distance from the centre of the first gear to its stationary pivot

$r_{c n} \quad$ distance from the centre of the last gear to its sliding pivot

$\beta \quad$ overall gear ratio

$D_{1} \quad$ pitch circle diameter of the first gear

$D_{n} \quad$ pitch circle diameter of the last gear

$n \quad$ the number of gears on the arm

$\theta \quad$ the angular displacement of the first gear (i.e. the crank)

$\theta_{s} \quad$ the angular position of the crank at the assembly position

$\psi \quad$ the angular displacement of the last gear

$\psi_{s} \quad$ the angular position of the last gear at the assembly position

$\mu \quad$ the angular displacement of the arm

$\mu_{s} \quad$ the angular position of the arm at the assembly position

$t$ time

$x \quad$ the $\mathrm{x}$-coordinate of the slider (variable)

$h \quad$ the y-coordinate of the slider (constant)

$\sigma \quad$ the number of crank rotations per cycle

$i \quad$ step counter (used for the numerical position solution)

$J_{\theta i} \quad$ kinematic Jacobian at step number $i$

$J_{x i} \quad$ another kinematic Jacobian at step number $i$

$q \quad$ counter for points on the guiding curve

$f_{x q} \quad$ objective functions at point number $q$ 
$x g_{q} \quad \mathrm{X}$-coordinate of point number $q$ on the guiding curve

$N \quad$ total number of points on the guiding curve

j counter for precision points

$M \quad$ total number of precision points

$g_{v j} \quad$ constraint equations for the velocities at the precision points

$g_{m} \quad$ constraint equation for mobility

$g_{h} \quad$ constraint equation for the distance $h$

$g_{c 1} \quad$ constraint equation for the length $r_{c 1}$

$g_{c n} \quad$ constraint equation for the length $r_{c n}$

$g_{l} \quad$ constraint equation for the length $l_{a}$

$a_{m} \quad$ slack variable associated with $g_{m}$

$a_{h} \quad$ slack variable associated with $g_{h}$

$a_{c 1} \quad$ slack variable associated with $g_{c 1}$

$a_{c n} \quad$ slack variable associated with $g_{c n}$

$a_{l} \quad$ slack variable associated with $g_{l}$

$h_{\max }$ maximum allowable value for the distance $h$

$l_{a \min } \quad$ minimum allowable value for the arm length

$r_{c 1 \text { min }}$ minimum allowable value for the crank length

$r_{c n \text { min }}$ minimum allowable value for the length $r_{c n}$

$k \quad$ counter for the optimisation iterations

v design vector

$\delta \mathbf{v}$ design parameter correction vector

$\delta \mathbf{v}_{k} \quad$ correction vector at iteration number $k$

e error vector

$\mathbf{e}_{k} \quad$ error vector at iteration number $k$

J system Jacobian

$\mathbf{J}_{k} \quad$ system Jacobian at iteration number $k$ 
W weighting matrix

I identity matrix

$\lambda$ damping factor

$\varepsilon \quad$ allowable error value

\section{REFERENCES}

[1] Gabriele, G. A., 1993, "Optimization in Mechanisms, in Erdman, A. (Ed.), Modern Kinematics in the Last Forty Years", John Wiley \& Son Inc. New York.

[2] Zhang, X., Zhou, J. and Ye, Y. 1998, “Optimal Mechanism Design Using Interior-Points Methods", Mech. Mach. Theory, Vol. 35, pp. 83-98

[3] Cossalter, V., Doria, A., Pasini, M. and Scattolo, C., 1992, "A Simple Numerical Approach for Optimum Synthesis of a Class of Planar Mechanisms”, Mech. Mach. Theory, Vol. 27 (3), pp. 357-366.

[4] Minnaar, R. J., Tortorelli, D. A. and Snyman, J. A., 2001, “On Nonassembly in the Optimal Dimensional Synthesis of Planar Mechanisms", Struct. Multidisc. Optim., Vol. 1, pp. 345-354.

[5] Jensen, O. F. and Hansen, J. M., 2006, "Dimensional Synthesis of Spatial Mechanisms and the Problem of Non-Assembly", Multibody System Dynamics, No. 15 , pp. 1207-133.

[6] Hansen, J. M., 2002, "Synthesis of Mechanisms Using Time-Varying Dimensions”, Multibody System Dynamics, No. 2, pp. 127-144.

[7] Deb, K. and Tiwari, S., 2005, "Mutli-Objective Optimization of a Leg Mechanism Using Genetic Algorithms”, Eng. Optim., Vol. 37 (4), pp. 325-350.

[8] Cabrera, J. A., Simon, A. and Prado, M., 2002, "Optimal Synthesis of Mechanisms with Genetic Algorithms", Mech. Mach. Theory, Vol. 37, pp. 11651177.

[9] Smaili, A. A., Diab, N. A. and Atallah, N. A., 2005, “Optimum Synthesis of Mechanisms Using Tabu-Gradient Search Algorithm”, ASME J. Mech. Des., Vol. 127 (2), pp. 917-923. 
[10] Dooner, D. B. 2001, "Function Generation Utilizing and Eight-Link Mechanism and Optimized Non-Circular Gear Elements with Application to Automotive Steering”, Proc. Instn Mech. Engrs, Part C: J. Mechanical Engineering Science, Vol. 215 (C7), pp. 847-857.

[11] Nokleby, S. B. and Podhorodeski, R. P., 2001, "Optimization-Based Synthesis of Grashof Geared Five-Bar Mechanisms", ASME J. Mech. Des., Vol. 123 (12), pp. 529-533.

[12] Suchora, D. H. and Savage, M., 1974a, "Geared Five-Bar Crank Mechanism Part I: Analysis, Design Engineering Technical Conference", New York, Oct 5-9.

[13] Suchora, D. H. and Savage, M., 1974b, "Geared Five-Bar Crank Mechanism Part II: Design, Design Engineering Technical Conference", New York, Oct 5-9.

[14] Bresland, C., 2001, "Coupling Arrangement for Reciprocating Piston Engine", USA Patent: 6,273,052 B1.

[15] Norton, R. L., 1999, "Design of Machinery- An Introduction to the Synthesis and Analysis of Mechanisms and Machines", McGraw-Hill, New York.

[16] Haug, E.J., 1989, "Computer Aided Kinematics and Dynamics of Mechanical Systems - Volume 1: Basic Methods", Allyn and Bacon, Massachusetts.

[17] Antoniou, A. and Lu, W.-S., 2007, "Practical Optimization: Algorithms and Engineering Applications", Springer Science + Business Media, LLC, New York. 


\section{List of Figures: (6 in Total)}

Figure 1. A schematic drawing of the geared five-bar slider-crank mechanism

Figure 2. The two Types found for the geared five-bar slider-crank mechanism

Figure 3. The concept of guiding curve

Figure 4. A summary of the computational approach used in the paper

Figure 5. Slider trajectories for case study 1

Figure 6. Slider trajectories for case study 2

\section{List of Tables: (2 in Total)}

Table 1. The desired stroke particulars for Case Study 1

Table 2. The desired stroke particulars for Case Study 2 
- 21 - 


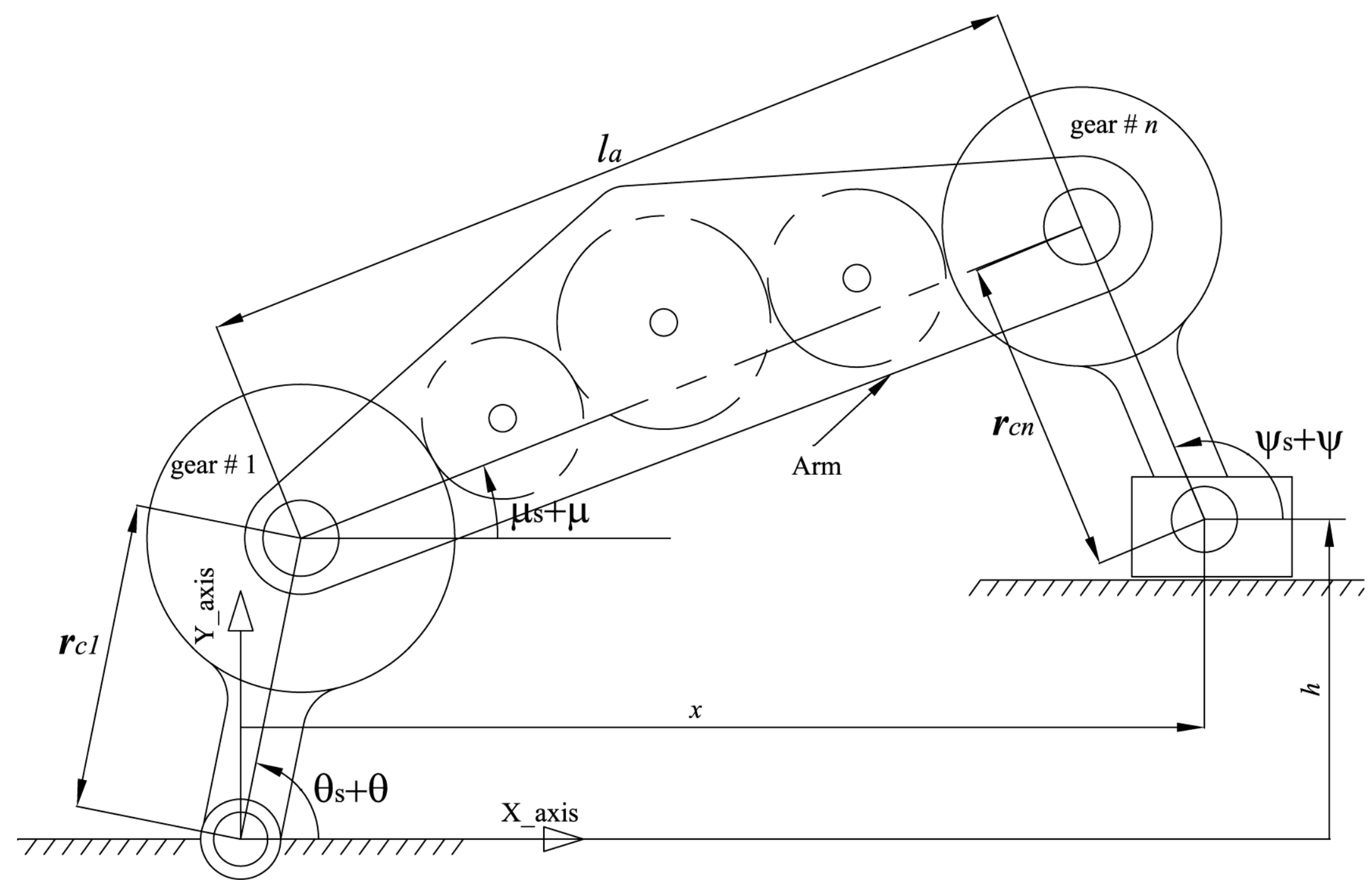

Figure 1. A schematic drawing of the geared five-bar slider-crank mechanism 

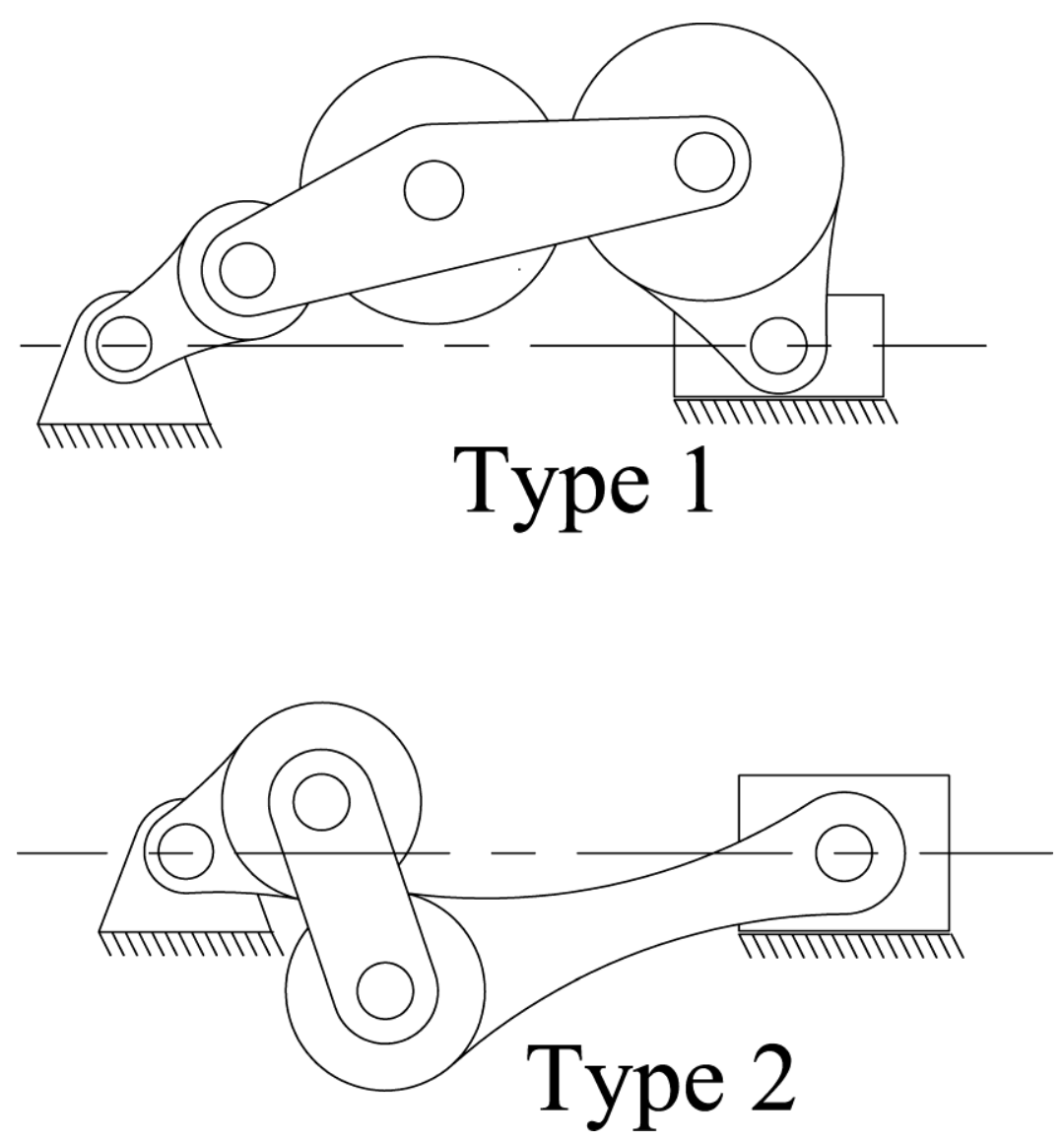

Figure 2. The two Types found for the geared five-bar slider-crank mechanism 


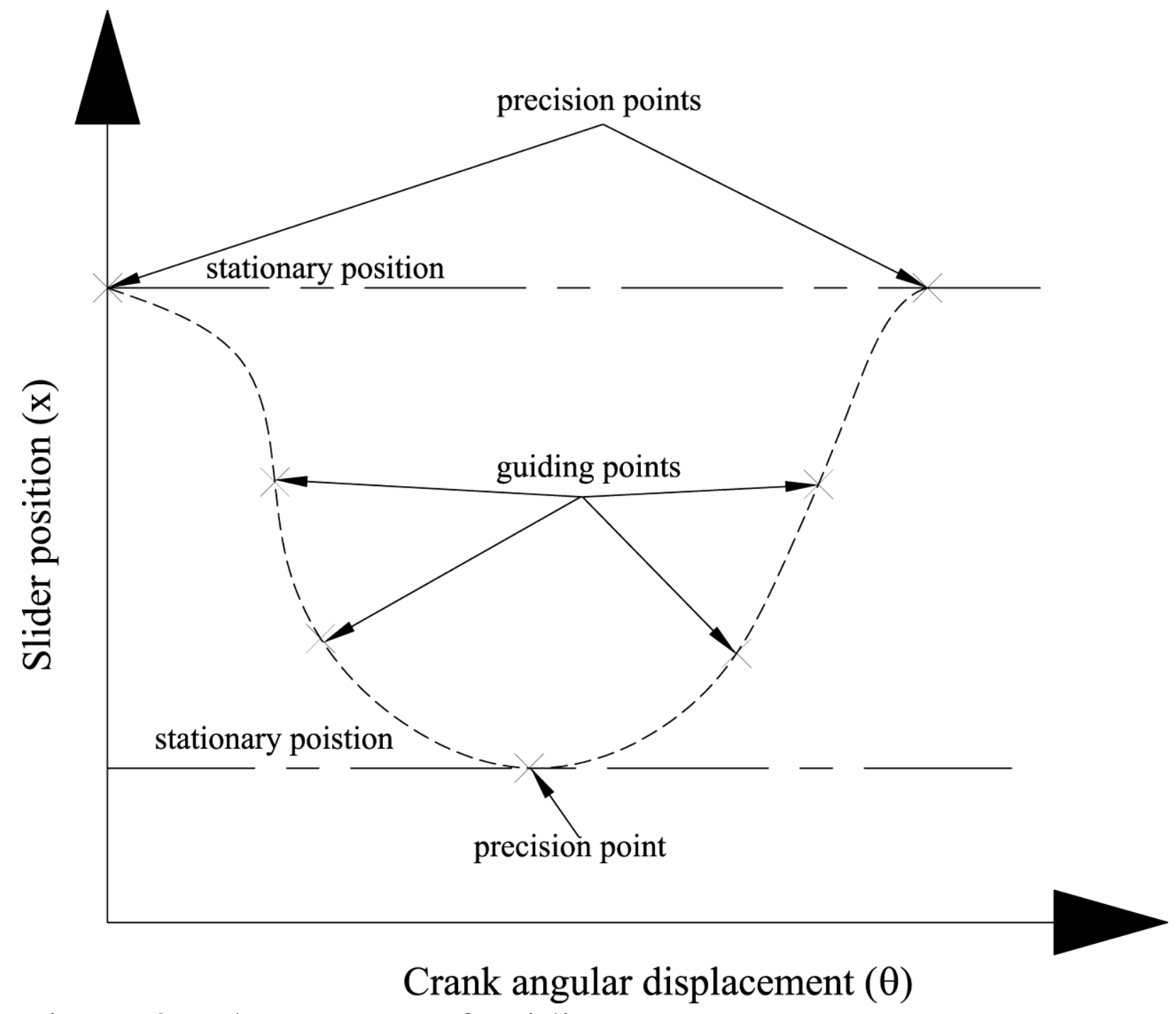

Figure 3. The concept of guiding curve 


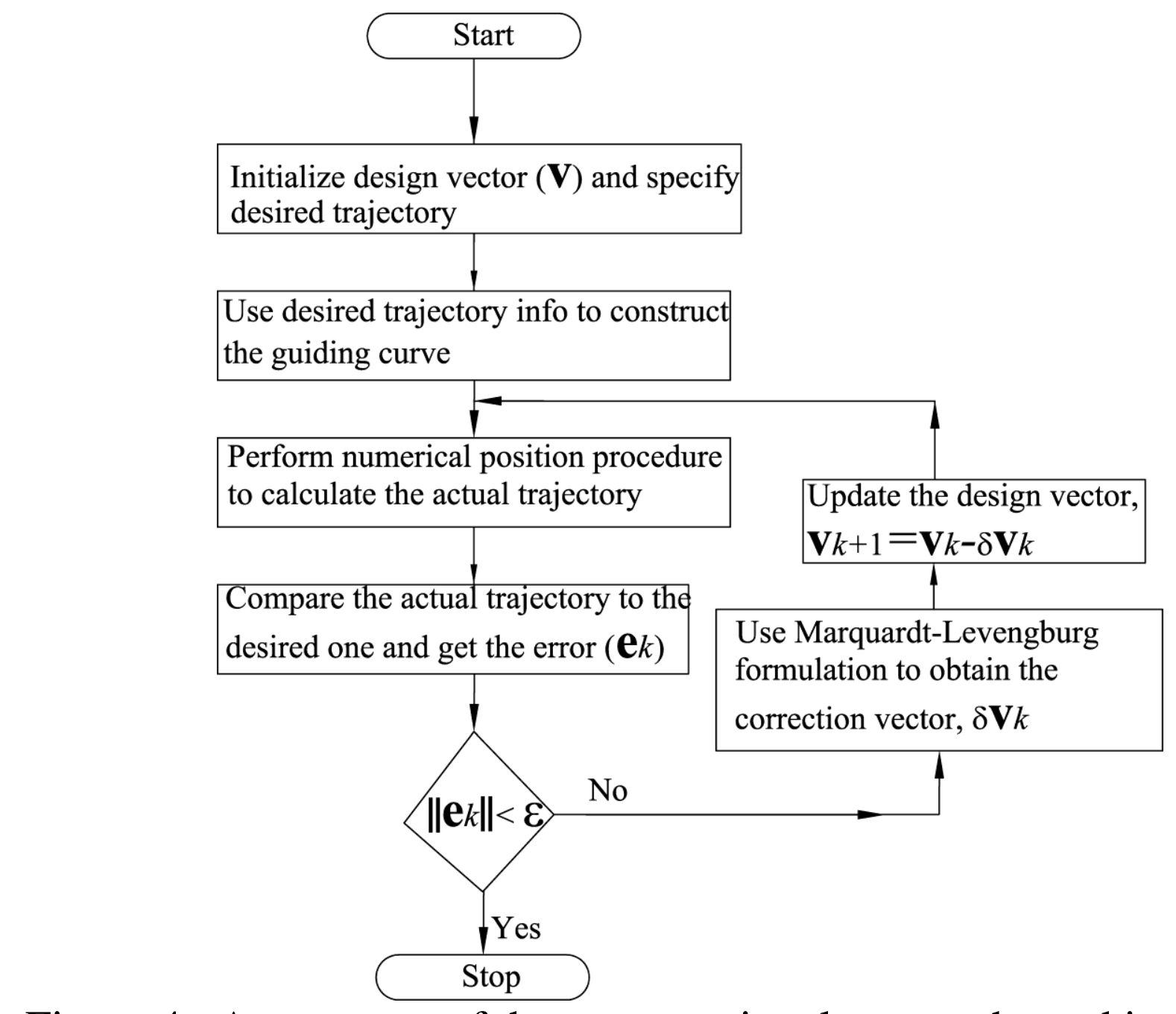

Figure 4. A summary of the computational approach used in the paper. 


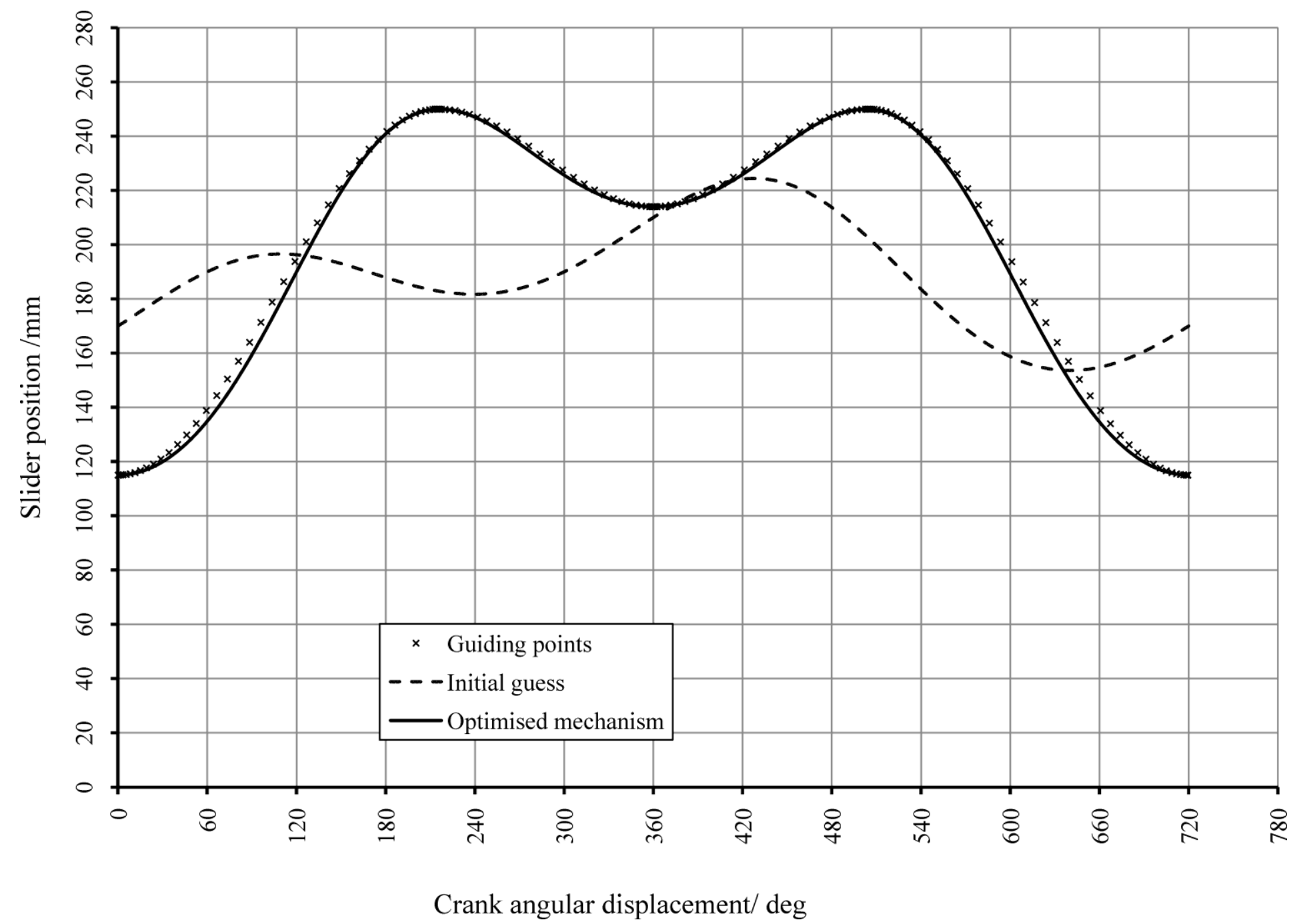

Figure 5. Slider trajectories for case study 1 


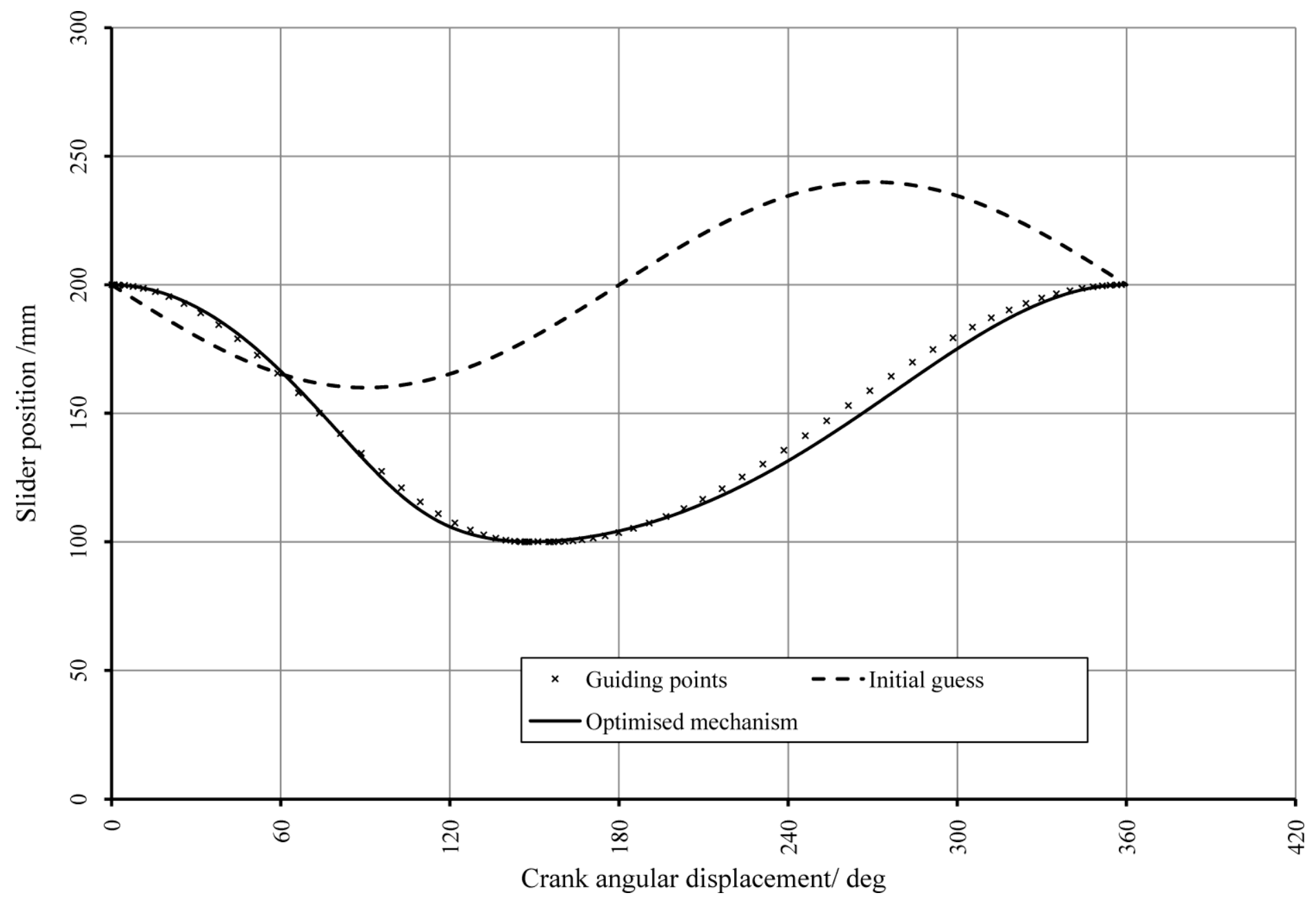

Figure 6. Slider trajectories for case study 2 
Table 1. The desired stroke particulars for Case Study 1

\begin{tabular}{|l|c|c|c|c|}
\hline Stroke Number & 1 & 2 & 3 & 4 \\
\hline x-coordinate at start of stroke (mm) & 114 & 250 & 214 & 250 \\
\hline x-coordinate at end of stroke (mm) & 250 & 214 & 250 & 114 \\
\hline Corresponding crank rotation $(\mathrm{deg})$ & 215 & 145 & 145 & 215 \\
\hline
\end{tabular}

Table 2. The desired stroke particulars for Case Study 2

\begin{tabular}{|l|c|c|}
\hline Stroke Number & 1 & 2 \\
\hline$x$-coordinate at start of stroke $(\mathrm{mm})$ & 100 & 200 \\
\hline $\mathrm{x}$-coordinate at end of stroke (mm) & 200 & 100 \\
\hline Corresponding crank rotation (deg) & 147.5 & 205 \\
\hline
\end{tabular}

